\title{
REFINEMENTS OF THE HERMITE-HADAMARD INEQUALITY IN NPC GLOBAL SPACES
}

\author{
Cristian Conde
}

Abstract. In this paper we establish different refinements and applications of the Hermite-Hadamard inequality for convex functions in the context of NPC global spaces.

\section{Introduction}

Recall that $f:[a, b] \rightarrow \mathbb{R}$ is called a convex function if it holds

$$
f(\lambda x+(1-\lambda) y) \leq \lambda f(x)+(1-\lambda) f(y)
$$

for all $x, y \in[a, b]$ and $0 \leq \lambda \leq 1$. It is well known that every convex function can be modified at the endpoints to become convex and continuous. An immediate consequence of this remark is the integrability of $f$. The integral of $f$ can then be estimated by:

$$
f\left(\frac{a+b}{2}\right) \leq \frac{1}{b-a} \int_{a}^{b} f(t) d t \leq \frac{f(a)+f(b)}{2} .
$$

This fundamental inequality is well known as the Hermite-Hadamard $(\mathrm{H}-\mathrm{H})$ inequality, it was first published by Hermite in 1883 and independently proved

Received: 21.04.2017. Accepted: 21.09.2017. Published online: 31.01.2018.

(2010) Mathematics Subject Classification: 39B62, 32F17, 26E60.

Key words and phrases: nonpositive curvature metric space, geodesic convexity, short geodesic, Hermite-Hadamard inequality, means. 
in 1893 by Hadamard in [12]. One can easily observe that (1.1) is equivalent to the following inequality:

$$
f\left(\frac{a+b}{2}\right) \leq \int_{0}^{1} f(t a+(1-t) b) d t \leq \frac{f(a)+f(b)}{2} .
$$

From the previous statement, we have concluded in [7] that the notions or concepts necessaries to obtain an analogue of the $\mathrm{H}-\mathrm{H}$ inequality in a global NPC space $N$ are: the existence of an unique geodesic connecting two given points, the notion of convex function associated with this privileged curve and convex sets. Since these concepts exist in the context of global NPC spaces, we obtained a generalization of the $\mathrm{H}-\mathrm{H}$ inequality to such spaces.

The Hermite-Hadamard inequality has several applications. We would like to refer the reader to [10, 2, 16, 21, 19, 13, 1, 22, 6, 3] and references therein for more information. In this paper we refine one side of $\mathrm{H}-\mathrm{H}$ inequality in NPC global spaces via sequences.

\section{Preliminaries}

The aim of our paper is to discuss refinements of the $\mathrm{H}-\mathrm{H}$ inequality to the framework of spaces with global nonpositive curvature. A formal definition of these spaces is as follows.

Definition 2.1. Let $(N, d)$ be a complete metric space, which is also a geodesic length space in the sense that the distance of $N$ can be computed via the infimum of the length of the rectifiable arcs joining given endpoints in $N$ (see [14, Section 2.2]). We say that $(N, d)$ is a global $N P C$ space if for $x_{1}, x_{2} \in N$ there exists a point $z \in N$ such that for each $x \in N$ we have

$$
d^{2}(x, z) \leq \frac{1}{2} d^{2}\left(x, x_{1}\right)+\frac{1}{2} d^{2}\left(x, x_{2}\right)-\frac{1}{4} d^{2}\left(x_{1}, x_{2}\right) .
$$

These spaces are also known as the Cat $(0)$ spaces. In a global NPC space each pair of points $x_{1}, x_{2} \in N$ can be connected by a geodesic, i.e. by a rectifiable curve $\gamma:[0,1] \rightarrow N$ such that the length of $\left.\gamma\right|_{[s, t]}$ is $d(\gamma(s), \gamma(t))$ for all $0 \leq s \leq t \leq 1$. Moreover, this geodesic is unique. The point $z$ that appears in Definition 2.1 is the midpoint of $x_{1}$ and $x_{2}$ and has the property

$$
d\left(x_{1}, z\right)=d\left(x_{2}, z\right)=\frac{1}{2} d\left(x_{1}, x_{2}\right) .
$$


ExAmPle 2.2. (1) Every Hilbert space is a global NPC space. Its geodesics are the line segments.

(2) A Riemannian manifold $(M, g)$ is a global NPC space if and only if it is complete, simply connected, and of nonpositive sectional curvature.

(3) Let $\operatorname{Sym}^{+}(n, \mathbb{R})$ be the set of all $n \times n$ dimensional positive definite matrices with real coefficients and for $A, B \in \operatorname{Sym}^{+}(n, \mathbb{R})$ we consider the metric

$$
d(A, B)=\left(\sum_{k} \log ^{2} \lambda_{k}\right)^{1 / 2},
$$

where $\lambda_{1}, \ldots, \lambda_{n}$ are the eigenvalues of $A B^{-1}$. Then $\left(\operatorname{Sym}^{+}(n, \mathbb{R}), d\right)$ is a global NPC space (see [17]).

Definition 2.3. A subset $C \subseteq N$ is called convex if for each geodesic $\gamma:[0,1] \rightarrow N$ joining two arbitrary points in $C$ holds that $\gamma([0,1]) \subseteq C$.

A function $f: C \rightarrow \mathbb{R}$ is called convex if the function $f \circ \gamma:[0,1] \rightarrow \mathbb{R}$ is convex whenever $\gamma:[0,1] \rightarrow C$ is geodesic, that is, for all $t \in[0,1]$

$$
f(\gamma(t)) \leq(1-t) f(\gamma(0))+t f(\gamma(1)) .
$$

For sake of completeness, we recall two inequalities obtained in a previous work (see [7]). These statements formulate different refinements of the $\mathrm{H}-\mathrm{H}$ inequality in global NPC spaces.

Theorem 2.4 ([7, Theorem 3.3]). Let $(N, d)$ be a global NPC space, $C \subseteq N$ a convex set and $f: C \rightarrow \mathbb{R}$ a convex function. Then

$$
\begin{aligned}
f(\gamma(1 / 2)) & \leq \frac{1}{2}[f(\gamma(2 / 3))+f(\gamma(1 / 3))] \leq \frac{1}{2}[f(\gamma(1 / 4))+f(\gamma(3 / 4))] \\
& \leq \int_{0}^{1} f(\gamma(t)) d t \\
& \leq \frac{1}{2}\left[f(\gamma(1 / 2))+\frac{f(\gamma(0))+f(\gamma(1))}{2}\right] \leq \frac{f(\gamma(0))+f(\gamma(1))}{2}
\end{aligned}
$$

and

$$
f(\gamma(1 / 2)) \leq l(\lambda) \leq \int_{0}^{1} f(\gamma(t)) d t \leq L(\lambda) \leq \frac{f(\gamma(0))+f(\gamma(1))}{2}
$$


for all geodesic $\gamma:[0,1] \rightarrow C$ and $\lambda \in[0,1]$, where

$$
l(\lambda)=\lambda f\left(\gamma\left(\frac{\lambda}{2}\right)\right)+(1-\lambda) f\left(\gamma\left(\frac{1+\lambda}{2}\right)\right)
$$

and

$$
L(\lambda)=\frac{1}{2}[f(\gamma(\lambda))+\lambda f(\gamma(0))+(1-\lambda) f(\gamma(1))]
$$

REMARK 2.5. Note that the following inequality is valid for a convex function $f: C \rightarrow \mathbb{R}$, where $C \subseteq N$ is a convex set:

$$
\int_{0}^{1} f(\gamma(t)) d t-l(\lambda) \leq L(\lambda)-\int_{0}^{1} f(\gamma(t)) d t
$$

The proof is a consequence of the bounds of the normalized Jensen functional (see [8, Corollary 1] or [20, Corollary 3.1]), more precisely: let $f$ be a convex function on $[a, b]$. Then

$$
\begin{aligned}
& 2 \lambda_{\min }\left(\frac{f\left(x_{1}\right)+f\left(x_{2}\right)}{2}-f\left(\frac{x_{1}+x_{2}}{2}\right)\right) \\
& \leq \lambda f\left(x_{1}\right)+(1-\lambda) f\left(x_{2}\right)-f\left(\lambda x_{1}+(1-\lambda) x_{2}\right) \\
& \leq 2 \lambda_{\max }\left(\frac{f\left(x_{1}\right)+f\left(x_{2}\right)}{2}-f\left(\frac{x_{1}+x_{2}}{2}\right)\right),
\end{aligned}
$$

where $0<\lambda<1, \lambda_{\min }=\min \{\lambda, 1-\lambda\}, \lambda_{\max }=\max \{\lambda, 1-\lambda\}$ and $x_{1}, x_{2} \in[a, b]$.

\section{Refinements of Hermite-Hadamard inequality}

We begin this section with a statement that has motivated the other results obtained in this work.

Lemma 3.1. Let $f:[a, b] \rightarrow \mathbb{R}$ be a convex function. Then

$$
\begin{aligned}
f\left(\frac{a+b}{2}\right) & \leq \frac{1}{b-a} \int_{a}^{b} f(t) d t \leq \frac{1}{2}\left(f\left(\frac{a+b}{2}\right)+\frac{1}{2} f(a)+\frac{1}{2} f(b)\right) \\
& \leq \frac{1}{16}\left(2 f\left(\frac{a+b}{2}\right)+7 f(a)+7 f(b)\right) \leq \frac{f(a)+f(b)}{2}
\end{aligned}
$$


Proof. Using convexity of the function $f$, we can easily verify the last inequality. Now we will prove that:

$$
\begin{aligned}
\frac{1}{b-a} \int_{a}^{b} f(t) d t & \leq \frac{1}{2}\left(f\left(\frac{a+b}{2}\right)+\frac{1}{2} f(a)+\frac{1}{2} f(b)\right) \\
& \leq \frac{1}{16}\left(2 f\left(\frac{a+b}{2}\right)+7 f(a)+7 f(b)\right) .
\end{aligned}
$$

Let us note that the first inequality is well-known ([11, Theorem 1.1]), but we include its proof here for the sake of completeness. We have

$$
\begin{aligned}
\frac{1}{b-a} \int_{a}^{b} f(t) d t & \left.=\frac{1}{b-a}\left(\int_{a}^{\frac{a+b}{2}} f(t) d t+\int_{\frac{a+b}{2}}^{b} f(t) d t\right)\right) \\
& \leq \frac{1}{b-a}\left(\frac{f(a)+f\left(\frac{a+b}{2}\right)}{2} \frac{b-a}{2}+\frac{f(b)+f\left(\frac{a+b}{2}\right)}{2} \frac{b-a}{2}\right) \\
& =\frac{1}{4}\left(2 f\left(\frac{a+b}{2}\right)+f(a)+f(b)\right) \\
& =\frac{1}{16}\left(8 f\left(\frac{a+b}{2}\right)+4 f(a)+4 f(b)\right) \\
& \leq \frac{1}{16}\left(2 f\left(\frac{a+b}{2}\right)+3(f(a)+f(b))+4 f(a)+4 f(b)\right) \\
& =\frac{1}{16}\left(2 f\left(\frac{a+b}{2}\right)+7 f(a)+7 f(b)\right) .
\end{aligned}
$$

From now on in this section, we assume that $(N, d)$ is a global NPC space, $C \subseteq N$ a convex set, $f: C \rightarrow \mathbb{R}$ a convex function and $\gamma:[0,1] \rightarrow C$ a geodesic connecting $\gamma(0)$ with $\gamma(1)$. In this paper we establish a refinement of inequality (2.1). For this let us first define the following sequences (motivated by the previous Lemma 3.1 for any $n \in \mathbb{N}$ with $n \geq 2$ :

$$
x_{n}=\frac{1}{2}\left[f\left(\gamma\left(\frac{1}{n}\right)\right)+f\left(\gamma\left(1-\frac{1}{n}\right)\right)\right]
$$

and

$$
y_{n}=\frac{2}{n+1} f\left(\gamma\left(\frac{1}{2}\right)\right)+\left[1-\frac{2}{n+1}\right] \frac{f(\gamma(0))+f(\gamma(1))}{2} .
$$


REMARK 3.2. In the particular case of $\mathbb{R}$ with the usual distance we have

$$
y_{3}=\frac{1}{2}\left(f\left(\frac{a+b}{2}\right)+\frac{1}{2} f(a)+\frac{1}{2} f(b)\right)
$$

and

$$
y_{15}=\frac{1}{16}\left(2 f\left(\frac{a+b}{2}\right)+7 f(a)+7 f(b)\right) .
$$

The following result is a refinement of the "arithmetic-geometric" inequality.

Proposition 3.3. Let $x_{n}$ and $y_{n}$ be sequences defined as above. Then

(i) $\left\{x_{n}\right\}_{n \geq 2}$ and $\left\{y_{n}\right\}_{n \geq 2}$ are non-decreasing sequences,

(ii) for $n \geq 2$ we have

$$
f(\gamma(1 / 2)) \leq x_{n} \leq y_{n} \leq \frac{f(\gamma(0))+f(\gamma(1))}{2} .
$$

Proof. (i) The monotonicity of $\left\{x_{n}\right\}_{n \geq 2}$ is a consequence of the convexity of $f$ and the following relations:

$$
\gamma\left(\frac{1}{n}\right)=\gamma\left(\left[1-\frac{1}{n(n-1)}\right] \frac{1}{n+1}+\frac{1}{n(n-1)} \frac{n}{n+1}\right)
$$

and

$$
\gamma\left(1-\frac{1}{n}\right)=\gamma\left(\left[1-\frac{1}{n(n-1)}\right] \frac{n}{n+1}+\frac{1}{n(n-1)} \frac{1}{n+1}\right) .
$$

On the other hand, $\left\{y_{n}\right\}_{n \geq 2}$ is a non-decreasing sequence as consequence of (2.1).

(ii) The first inequality is a consequence of the convexity of $f$ and the fact that for any $t_{0} \in[0,1]$ the midpoint between $\gamma\left(t_{0}\right)$ and $\gamma\left(1-t_{0}\right)$ is given by $\gamma(1 / 2)$. Then in particular, if we consider $t_{0}=1 / n$, we obtain the relation

$$
f(\gamma(1 / 2)) \leq \frac{1}{2}\left[f\left(\gamma\left(\frac{1}{n}\right)\right)+f\left(\gamma\left(1-\frac{1}{n}\right)\right)\right] .
$$

To prove the second inequality, we use the fact that $\left\{x_{n}\right\}_{n \geq 2}$ is a non-decreasing sequence, the convexity of $f$ and the following equalities:

$$
\gamma\left(\frac{1}{n+1}\right)=\gamma\left(\left[1-\frac{2}{n+1}\right] 0+\frac{2}{n+1} \frac{1}{2}\right),
$$




$$
\gamma\left(\frac{n}{n+1}\right)=\gamma\left(\left[1-\frac{2}{n+1}\right]+\frac{2}{n+1} \frac{1}{2}\right)
$$

Finally, the last inequality is a consequence of 2.1 .

With the sequences introduced above, we can rewrite the inequality 2.1 as follows:

$$
f(\gamma(1 / 2))=x_{2} \leq x_{3} \leq x_{4} \leq \int_{0}^{1} f(\gamma(t)) d t \leq y_{3} \leq \frac{f(\gamma(0))+f(\gamma(1))}{2}
$$

From the previous statement, we obtain the following improvement of the $\mathrm{H}-\mathrm{H}$ inequality and refinement of Lemma 3.1 .

TheOrem 3.4. Let $(N, d)$ be a global NPC space, $C \subseteq N$ a convex set, $f: C \rightarrow \mathbb{R}$ a convex function and $n \geq 3$. Then

$$
\begin{aligned}
f(\gamma(1 / 2)) & \leq x_{3} \leq x_{4} \leq \int_{0}^{1} f(\gamma(\lambda)) d \lambda \leq y_{3} \leq \ldots y_{n} \leq \ldots \\
& \leq \frac{f(\gamma(0))+f(\gamma(1))}{2}
\end{aligned}
$$

for all geodesic $\gamma:[0,1] \rightarrow C$.

Now we consider the following sequences, for $n \geq 2$ :

$$
z_{n}=\frac{1}{2}\left(x_{n}+\frac{f(\gamma(0))+f(\gamma(1))}{2}\right)
$$

and

$$
w_{n}=\frac{1}{2}\left(y_{n}+\frac{f(\gamma(0))+f(\gamma(1))}{2}\right)
$$

Proposition 3.5. Let $z_{n}, w_{n}$ be the sequences defined above. Then

(i) $\left\{z_{n}\right\}_{n \geq 2}$ and $\left\{z_{n}\right\}_{n \geq 2}$ are non-decreasing sequences,

(ii) for $n \geq 2$ we have $z_{n} \leq w_{n}$,

(iii) for $n \geq 2$ we have

$$
\begin{aligned}
\frac{1}{2}\left[f(\gamma(1 / 2))+\frac{f(\gamma(0))+f(\gamma(1))}{2}\right] & =z_{2} \leq \ldots \leq z_{n} \leq \ldots \\
& \leq \frac{f(\gamma(0))+f(\gamma(1))}{2}
\end{aligned}
$$


and for $n \geq 3$

$$
\begin{aligned}
\frac{1}{2}\left[f(\gamma(1 / 2))+\frac{f(\gamma(0))+f(\gamma(1))}{2}\right] & =w_{3} \leq \ldots \leq w_{n} \leq \ldots \\
& \leq \frac{f(\gamma(0))+f(\gamma(1))}{2} .
\end{aligned}
$$

Proof. It is a consequence of Proposition 3.3 and the fact that $z_{2}=$ $y_{3}=w_{3}$.

TheOrem 3.6. Let $(N, d)$ be a global $N P C$ space, $C \subseteq N$ a convex set and $f: C \rightarrow \mathbb{R}$ a convex function. Then for $n \geq 2$

$$
\begin{aligned}
f(\gamma(1 / 2)) & \leq x_{3} \leq x_{4} \leq \int_{0}^{1} f(\gamma(\lambda)) d \lambda \leq z_{2} \leq \ldots z_{n} \leq \ldots \\
& \leq \frac{f(\gamma(0))+f(\gamma(1))}{2}
\end{aligned}
$$

and for $m \geq 3$

$$
\begin{aligned}
f(\gamma(1 / 2)) & \leq x_{3} \leq x_{4} \leq \int_{0}^{1} f(\gamma(\lambda)) d \lambda \leq w_{3} \leq \ldots w_{m} \leq \ldots \\
& \leq \frac{f(\gamma(0))+f(\gamma(1))}{2}
\end{aligned}
$$

for all geodesic $\gamma:[0,1] \rightarrow C$.

\section{Applications}

4.1. Let us consider $(N, d)=(\mathbb{R},|\cdot|)$, the global NPC space of the real numbers with the usual distance, i.e. $d(x, y)=|x-y|$. If we take $\gamma_{a, b}(t)=$ $(1-t) a+t b$, the unique geodesic connecting $a$ to $b$, and $f:[a, b] \rightarrow \mathbb{R}$ a convex function, then:

$$
\begin{aligned}
& x_{n}=\frac{1}{2}\left[f\left(\frac{a+(n-1) b}{n}\right)+f\left(\frac{(n-1) a+b}{n}\right)\right], \\
& y_{n}=\frac{2}{n+1} f\left(\frac{a+b}{2}\right)+\left[1-\frac{2}{n+1}\right] \frac{f(a)+f(b)}{2},
\end{aligned}
$$




$$
\begin{aligned}
z_{n} & =\frac{1}{2}\left(x_{n}+\frac{f(a)+f(b)}{2}\right), \\
w_{n} & =\frac{1}{2}\left(y_{n}+\frac{f(a)+f(b)}{2}\right) .
\end{aligned}
$$

In conclusion, we can state the following.

Proposition 4.1. Let $f:[a, b] \rightarrow \mathbb{R}$ be a convex function.

(1) For any $n \geq 3$ we have

$$
\begin{aligned}
f\left(\frac{a+b}{2}\right) & \leq x_{3} \leq x_{4} \leq \frac{1}{b-a} \int_{a}^{b} f(t) d t \leq \frac{1}{2}\left[f\left(\frac{a+b}{2}\right)+\frac{f(a)+f(b)}{2}\right] \\
& =y_{3} \leq \ldots \leq y_{n} \leq \ldots \leq \frac{f(a)+f(b)}{2}
\end{aligned}
$$

(2) For any $n \geq 2$ we have

$$
\begin{aligned}
f\left(\frac{a+b}{2}\right) & \leq x_{3} \leq x_{4} \leq \frac{1}{b-a} \int_{a}^{b} f(t) d t \leq \frac{1}{2}\left[f\left(\frac{a+b}{2}\right)+\frac{f(a)+f(b)}{2}\right] \\
& =z_{2} \leq \ldots \leq z_{n} \leq \ldots \leq \frac{f(a)+f(b)}{2}
\end{aligned}
$$

(3) For any $n \geq 3$ we have

$$
\begin{aligned}
f\left(\frac{a+b}{2}\right) & \leq x_{3} \leq x_{4} \leq \frac{1}{b-a} \int_{a}^{b} f(t) d t \leq \frac{1}{2}\left[f\left(\frac{a+b}{2}\right)+\frac{f(a)+f(b)}{2}\right] \\
& =w_{3} \leq \ldots \leq w_{n} \leq \ldots \leq \frac{f(a)+f(b)}{2}
\end{aligned}
$$

REMARK 4.2. We can compare the sequences $\left\{y_{n}\right\}_{n \geq 2},\left\{z_{n}\right\}_{n \geq 2}$ introduced above with the refinement given by Zabandan in [23]. Recall that the sequence introduced by him was defined as follows:

$$
\mathcal{Y}_{n}(f, a, b)=\frac{1}{2^{n}}\left[\frac{f(a)+f(b)}{2}+\sum_{i=1}^{2^{n}-1} f\left(\left(1-\frac{i}{2^{n}}\right) a+\frac{i}{2^{n}} b\right)\right]
$$

It is obvious that $\mathcal{Y}_{1}(f, a, b)=y_{3}=z_{2}$. Then, by the monotonicity of each of these sequences, we have that $\mathcal{Y}_{1}(f, a, b)$ is a lower bound and consequently 
we have the following refinement of the $\mathrm{H}-\mathrm{H}$ inequality:

$$
\begin{aligned}
f\left(\frac{a+b}{2}\right) & \leq x_{3} \leq x_{4} \leq \frac{1}{b-a} \int_{a}^{b} f(t) d t \leq \mathcal{Y}_{m}(f, a, b) \leq \ldots \leq \mathcal{Y}_{1}(f, a, b) \\
& =\frac{1}{2}\left[f\left(\frac{a+b}{2}\right)+\frac{f(a)+f(b)}{2}\right]=y_{3} \leq \ldots \leq y_{n} \leq \ldots \\
& \leq \frac{f(a)+f(b)}{2}
\end{aligned}
$$

for all $m \geq 1$ and $n \geq 3$.

\subsection{Classical means}

Recall that for two positive numbers $a$ and $b$ the following classical means are defined by:

$$
\begin{aligned}
A & =A(a, b)=\frac{a+b}{2} \quad \text { arithmetic mean, } \\
F_{\nu} & =F_{\nu}(a, b)=(1-\nu) \sqrt{a b}+\nu \frac{a+b}{2}, \nu \in[0,1] \quad \text { Heinz mean, } \\
H_{\nu} & =H_{\nu}(a, b)=\frac{a^{\nu} b^{1-\nu}+a^{1-\nu} b^{\nu}}{2}, \nu \in[0,1] \quad \text { Heron mean, } \\
G & =G(a, b)=\sqrt{a b} \quad \text { geometric mean, } \\
L & =L(a, b)=\frac{b-a}{\ln (b)-\ln (a)} \quad(a \neq b) ; \quad L(a, a)=a \text { logarithmic mean. }
\end{aligned}
$$

Combining the different statements obtained previously we get:

Proposition 4.3. For $\alpha, \beta>0$ we have

$$
G(\alpha, \beta) \leq F_{1 / n}(\alpha, \beta) \leq H_{1-\frac{2}{n+1}}(\alpha, \beta) \leq A(\alpha, \beta),
$$

in particular, if $\alpha \neq \beta$, then

$$
\begin{aligned}
G(\alpha, \beta) & <H_{1 / 3}(\alpha, \beta)<H_{1 / 4}(\alpha, \beta)<L(\alpha, \beta)<\left(\frac{\sqrt{\alpha}+\sqrt{\beta}}{2}\right)^{2} \\
& <H_{1-\frac{2}{n+1}}(\alpha, \beta)<A(\alpha, \beta),
\end{aligned}
$$




$$
\begin{aligned}
G(\alpha, \beta) & <H_{1 / 3}(\alpha, \beta)<H_{1 / 4}(\alpha, \beta)<L(\alpha, \beta)<\left(\frac{\sqrt{\alpha}+\sqrt{\beta}}{2}\right)^{2} \\
& <\frac{1}{2}\left(H_{1 / n}(\alpha, \beta)+A(\alpha, \beta)\right)<A(\alpha, \beta) \\
G(\alpha, \beta) & <H_{1 / 3}(\alpha, \beta)<H_{1 / 4}(\alpha, \beta)<L(\alpha, \beta)<\left(\frac{\sqrt{\alpha}+\sqrt{\beta}}{2}\right)^{2} \\
& <H_{1-\frac{1}{n+1}}(\alpha, \beta)<A(\alpha, \beta) .
\end{aligned}
$$

Proof. Consider Propositions 3.3 and 4.1 with $f(t)=e^{t}$ and $\alpha=e^{a}$, $\beta=e^{b}$.

The above proposition gives comparisons between Heinz and Heron means. Some of the previous inequalities have been obtained by Bhatia in [4 and Carlson in [5]. On the other hand, in [18] Leach and Sholander showed that

$$
\sqrt[3]{G^{2} A}<L
$$

Particularly, as an application of 4.1 and the arithmetic-geometric's inequality one can deduce

$$
G<\sqrt[3]{G^{2} A}<L
$$

Using the previous proposition, we can refine that inequality as follows:

$$
G<H_{1 / 3}(\alpha, \beta)<\sqrt[3]{G^{2} A}<L .
$$

4.3. Utilizing the previous refinement obtained for convex real functions defined in $[a, b]$ and mimicking the proof given by Dragomir in [9], we can provide some Hermite-Hadamard's type inequalities for operator convex functions of selfadjoint operators in Hilbert spaces.

\section{References}

[1] Abramovich S., Barić J., Pečarić J., Fejer and Hermite-Hadamard type inequalities for superquadratic functions, J. Math. Anal. Appl. 344 (2008), no. 2, 1048-1056.

[2] Barnett N.S., Cerone P., Dragomir S.S., Some new inequalities for Hermite-Hadamard divergence in information theory, Stochastic analysis and applications Vol. 3, 7-19, Nova Sci. Publ., Hauppauge, New York, 2003.

[3] Bessenyei M., Hermite-Hadamard-type inequalities for generalized convex functions, J. Inequal. Pure Appl. Math. 9 (2008), no. 3, Art. 63, 51 pp.

[4] Bhatia R., The logarithmic mean, Resonance 13 (2008), no. 6, 583-594.

[5] Carlson B.C., The logarithmic mean, Amer. Math. Monthly 79 (1972), no. 6, 615-618. 
[6] Cerone P., Dragomir S.S., Mathematical inequalities. A perspective, CRC Press, Boca Raton, 2011.

[7] Conde C., A version of the Hermite-Hadamard inequality in a nonpositive curvature space, Banach J. Math. Anal. 6 (2012), no. 2, 159-167.

[8] Dragomir S.S., Bounds for the normalised Jensen functional, Bull. Austral. Math. Soc. 74 (2006), no. 3, 471-478.

[9] Dragomir S.S., Hermite-Hadamard's type inequalities for operator convex functions, Appl. Math. Comput. 218 (2011), no. 3, 766-772.

[10] Dragomir S.S., Pearce C.E.M., Selected Topics on Hermite-Hadamard Inequalities, RGMIA Monographs, Victoria University, 2000. Available at http://rgmia.vu.edu. au/monographs/hermite_hadamard.html

[11] El Farissi A., Simple proof and refinement of Hermite-Hadamard inequality. J. Math. Inequal. 4 (2010), no. 3, 365-369.

[12] Hadamard J., Étude sur les propriétés des fonctions entières et en particulier d'une fonction considérée par Riemann (French), J. Math. Pures et Appl. 58 (1893), 171215.

[13] Házy A., Páles Z., On a certain stability of the Hermite-Hadamard inequality, Proc. R. Soc. Lond. Ser. A Math. Phys. Eng. Sci. 465 (2009), no. 2102, 571-583.

[14] Jost J., Nonpositive curvature: geometric and analytic aspects, Lectures in Mathematics ETH Zürich, Birkhäuser Verlag, Basel, 1997.

[15] Kikianty E., Hermite-Hadamard inequality in the geometry of Banach spaces, $\mathrm{PhD}$ thesis, Victoria University, 2010. Available at eprints.vu.edu.au/15793/1/ EderKikiantyThesis.pdf

[16] Klaričić M., Neuman E., Pečarić J., Šimić V., Hermite-Hadamard's inequalities for multivariate g-convex functions, Math. Inequal. Appl. 8 (2005), no. 2, 305-316.

[17] Lang S., Fundamentals of differential geometry, Graduate Texts in Mathematics, Springer-Verlag, New York, 1999.

[18] Leach E.B., Sholander M.C., Extended mean values. II, J. Math. Anal. Appl. 92 (1983), no. 1, 207-223.

[19] Mihăilescu M., Niculescu C.P., An extension of the Hermite-Hadamard inequality through subharmonic functions, Glasg. Math. J. 49 (2007), no. 3, 509-514.

[20] Mitroi F.-C., About the precision in Jensen-Steffensen inequality, An. Univ. Craiova Ser. Mat. Inform. 37 (2010), no. 4, 73-84.

[21] Niculescu C.P., Persson L.-E., Convex functions and their applications. A contemporary approach, Springer, New York, 2006.

[22] Wu S., On the weighted generalization of the Hermite-Hadamard inequality and its applications, Rocky Mountain J. Math. 39 (2009), no. 5, 1741-1749.

[23] Zabandan G., A new refinement of the Hermite-Hadamard inequality for convex functions, J. Inequal. Pure Appl. Math. 10 (2009), no. 2, Art. 45, 7 pp.

Instituto de Ciencias

Universidad Nacional de Gral. Sarmiento

J. M. Gutierrez 1150

(B1613GSX) Los Polvorines

AND

Instituto Argentino de Matemática "Alberto P. Calderón"

SAAVEDRA 15 3̌̃ PISO

(C1083ACA) Buenos Aires

Argentina

e-mail: cconde@ungs.edu.com 